\title{
Focal Segmental Glomerulosclerosis Collapsing Variant
}

National Cancer Institute

\section{Source}

National Cancer Institute. Focal Segmental Glomerulosclerosis Collapsing Variant. NCI

Thesaurus. Code C123052.

A variant of FSGS characterized by glomerular tuft collapse, which may result in scarring.

(D'Agati VD, et al. "Pathologic Classification of Focal Segmental Glomerulosclerosis: A Working Proposal." Am J Kidney Dis 43.2 (2004): 368-82.) 